\title{
NDF of the near-zone field on a line perpendicular to the source
}

\author{
ROCCO PIERRI ${ }^{1}$, RAFFAELE MORETTA ${ }^{1}$ (Student Member, IEEE) \\ ${ }^{1}$ Department of Engineering, University of Campania "Luigi Vanvitelli", 81031 Aversa, Italy \\ Corresponding author: Raffaele Moretta (e-mail: raffaele.moretta@unicampania.it).
}

This work was supported by the Italian Ministry of University and Research through the PON Initiative under the Project Leonardo 4.0 (Grant B26C18000080005).

\begin{abstract}
In this paper, the problem of computing the number of degrees of freedom (NDF) of the field radiated by a strip current along all the possible lines orthogonal to the source is addressed.

As well known, the NDF is equal to the number of singular values of the radiation operator that are before a critical index at which they abrupt decay. Unfortunately, in the considered case, the solution of the associate eigenvalue problem is not known in closed-form, and this prevents us from directly evaluating the singular values of the radiation operator.

To overcome this drawback, a weighted adjoint operator is exploited. The latter allows obtaining an eigenvalue problem whose solution is known in closed-form but, at the same time, it modifies the behavior of the singular values. However, since the change affects only the dynamics of the singular values but not the critical index at which they abrupt decay, the NDF of the radiated field can be analytically estimated by resorting to the weighted adjoint operator.
\end{abstract}

INDEX TERMS dimension of data, eigenvalue problem, inverse source problem, integral equation, radiation operator, singular values, NDF.

\section{INTRODUCTION}

$\mathbf{T}$ HE inverse source problem has several applications in antenna analysis and synthesis [1]-[9]. From the mathematical point of view, it entails inverting a linear integral operator called radiation operator. The latter relates the density current $J$ to the radiated field $E$ which represent respectively the unknown and the data of the inverse problem.

In order to evaluate the achievable performance in linear inverse problems, some common metrics are the point spread function (PSF) [10], and the number of degrees of freedom (NDF) [11]-[13]. In particular, the point spread function provides the reconstruction of an impulsive source or the synthesis of an impulsive field; instead, the number of degrees of freedom represents at the same time the number of independent functions required to represent the data with a given degree of accuracy and the dimension of the unknowns subspace that can be stably reconstructed [14].

Both the metrics depend on the geometric parameters of the configuration and both are linked to the singular values decomposition (SVD) of the radiation operator, which is a key mathematical tool widely used in electromagnetics [15][20].

The link between the singular values decomposition and the metrics mentioned above appears immediately clear considering that the point spread function can be expressed in terms of the singular system of the radiation operator while the number of degrees of freedom is given by the number of relevant singular values of such an operator [21] [22].

However, the SVD of the radiation operator can be exploited not only to evaluate the singular system, the number of degrees of freedom, and the point spread function [23]-[26] but also to find a discretization of the radiation operator that exploits a non-redundant number of samples [27]-[30].

In this article, a configuration consisting of a magnetic current strip observed in near-zone on a truncated line orthogonal to the source is dealt with. Such geometry has been considered in [31] [32] for the study of the depth resolution just in the case where the observation domain is centered with respect to the source. Differently, here, the focus is on the analytical estimation of the NDF; furthermore, it is studied also the case where the observation domain is not centered with respect to the source.

Let us remark that the study of the NDF for the configuration described above not only gives insight both on the forward and on inverse source problems but it is of great interest also in phase retrieval [33] when the phaseless data are collected on more scanning lines parallel to the source. In the latter context, the knowledge of the NDF along the direction orthogonal to the source allows establishing the number of scanning surfaces which provides independent information. In fact, taking into account that the square amplitude of the radiated field must be sampled with a step- 
length that is the half of the radiated field, it is possible to retain that the number of scanning surface which allows collecting independent data will be approximately equal to two times the NDF of the radiated field.

For the considered configuration, the kernel of the associate eigenvalue problem is space-variant; hence, the issue of finding the NDF by studying the singular values behavior of the radiation operator is quite difficult. To surmount this drawback, at first, a weighted adjoint operator is introduced and an asymptotic evaluation of the kernel of the eigenvalue is performed. After, by acting a change of variables, the original eigenvalue problem is recast in a new one.

If the observation domain is outside the source domain or centered with respect to it, the new eigenvalue problem involves a convolution operator with a sinc kernel. Hence, its eigenvalues can be computed by exploiting the closedform results related to the Slepian-Pollak operator [34] [35]. At this stage, the NDF of the radiated field will be obtained by observing that the presence of the weight function in the adjoint operator affects only the dynamics of the eigenvalues but it does not change the position of the knee; hence, the NDF corresponds to the number of relevant eigenvalues of the sinc kernel.

Differently, if the observation domain is in front of the source but not centered with respect to it, the kernel of the new eigenvalue problem can be expressed by a sinc function of difference type plus an error term that does not affect the number of relevant eigenvalues. As it will be shown, also in this case the presence of the weight function does not affect the position of the knee in the eigenvalues diagram. Hence, the NDF of the radiated field can be still evaluated by referring to the integral operator with the sinc kernel.

Consequently, our final result will be that of providing the NDF of the near-field for each possible observation domain orthogonal to the source.

The article is organized as follows. In section II, at first, the geometry of the problem is shown; after, the radiation operator and its adjoint are introduced. In section III, the expression of the kernel involved in the integral equation for the computation of the singular values of the radiation operator is deduced. In section IV and V, the above-mentioned kernel is evaluated respectively in the case where the observation domain is located in the region outside the source, and in the region in front of the source. Later, it is shown how it is possible to recast such kernel in a form more similar to a sinc kernel of difference type. In section VI, the number of degrees of freedom of the radiated field is found. A section of conclusion follows.

\section{GEOMETRY OF THE PROBLEM}

A magnetic current $\underline{J}(x)=J(x) \hat{i}_{y}$, supported on the set $S D=[-a, a]$ of the $x$-axis, radiates in a homogeneous medium with wavenumber $\beta$.

The electric field radiated by such current has two components: one along the $x$-axis, and another along the $z$-axis. The $x$ component of the electric field, $E$, is observed on

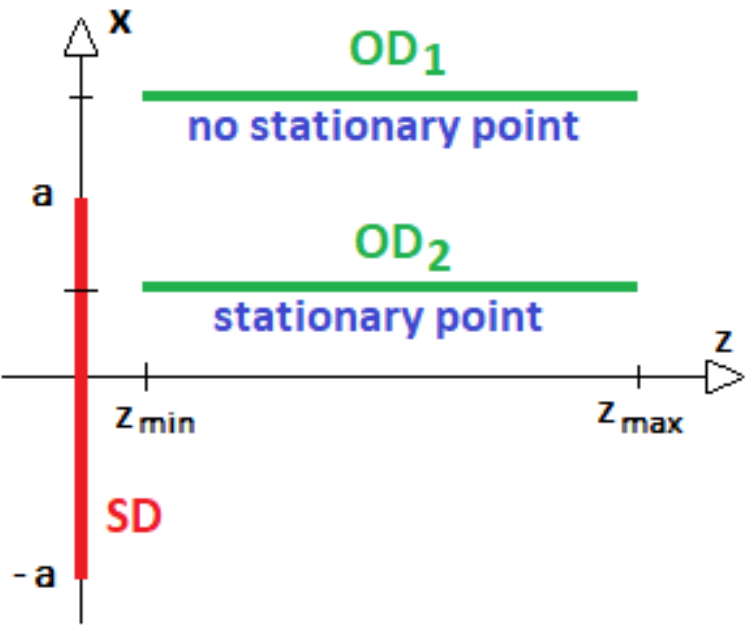

FIGURE 1. Geometry of the problem

a bounded observation domain $O D=\left[z_{\min }, z_{\max }\right]$ that is orthogonal to the source, and located in near non-reactive zone along the axis $x=x_{o}$ (see Fig. 1).

For the considered configuration, the radiation operator $\mathcal{T}$ can be expressed as below

$$
\mathcal{T} J=\int_{-a}^{a} z \frac{e^{-j \beta R\left(x^{\prime}, z\right)}}{R^{\frac{3}{2}}\left(x^{\prime}, z\right)} J\left(x^{\prime}\right) d x^{\prime}
$$

where

$$
R\left(x^{\prime}, z\right)=\sqrt{\left(x_{o}-x^{\prime}\right)^{2}+z^{2}} .
$$

The adjoint operator $\mathcal{T}^{\dagger}$ is usually defined as

$$
<\mathcal{T} J, E>_{L^{2}\left[z_{\min }, z_{\max }\right]}=\left\langle J, \mathcal{T}^{\dagger} E\right\rangle_{L^{2}[-a, a]}
$$

with $<,>_{L^{2}}$ denoting the scalar product in the functional space $L^{2}$ [36].

Here, instead, a weighted adjoint operator is introduced. The latter is given by

$$
\mathcal{T}_{w}^{\dagger} E=\mathcal{T}^{\dagger} w E=\int_{z_{\text {min }}}^{z_{\max }} w\left(x^{\prime}, z\right) z \frac{e^{j \beta R\left(x^{\prime}, z\right)}}{R^{\frac{3}{2}}\left(x^{\prime}, z\right)} E(z) d z
$$

where $w\left(x^{\prime}, z\right)$ denotes a real positive continuous function that pre-weights / pre-filters the data.

The use of the weighted adjoint has two effects. On one hand, it simplifies the mathematical analysis of the radiation operator. On the other, it changes the singular values decomposition of the radiation operator. The latter would appear a bad effect. However, since the weight function does not affect the phase of the kernel of the adjoint operator, its presence changes only the shape of the singular values but not the critical index at which they abrupt decay. For such reason, the weighted adjoint operator can be used in place of the usual adjoint for evaluating the NDF of the radiated field. 


\section{PRELIMINARIES CONCEPTS FOR THE STUDY OF THE RADIATION OPERATOR}

With the aim to study the singular values behavior of the radiation operator $\mathcal{T}$, the eigenvalues of the auxiliary operator $\mathcal{T} \mathcal{T}_{w}^{\dagger}$ will be studied. By virtue of the definitions (1) and (3), the latter can be expressed as

$$
\mathcal{T} \mathcal{T}_{w}^{\dagger} E=\int_{z_{\min }}^{z_{\max }} K\left(z_{o}, z\right) E(z) d z
$$

where

$$
K\left(z_{o}, z\right)=z_{o} z \int_{-a}^{a} w\left(x^{\prime}, z\right) \frac{e^{-j \beta\left[R\left(x^{\prime}, z_{o}\right)-R\left(x^{\prime}, z\right)\right]}}{R^{\frac{3}{2}}\left(x^{\prime}, z_{o}\right) R^{\frac{3}{2}}\left(x^{\prime}, z\right)} d x^{\prime}
$$

By setting $f\left(x^{\prime}, z_{o}, z\right)=1 /\left[R^{\frac{3}{2}}\left(x^{\prime}, z_{o}\right) R^{\frac{3}{2}}\left(x^{\prime}, z\right)\right]$ and $\phi\left(x^{\prime}, z_{o}, z\right)=\left[R\left(x^{\prime}, z_{o}\right)-R\left(x^{\prime}, z\right)\right] / a$, the kernel can be recast in the following form

$K\left(z_{o}, z\right)=z_{o} z \int_{-a}^{a} w\left(x^{\prime}, z\right) f\left(x^{\prime}, z_{o}, z\right) e^{-j \beta a \phi\left(x^{\prime}, z_{o}, z\right)} d x^{\prime}$

For $z_{o}=z$, the exponential function in (6) is equal to one; consequently, the kernel $K\left(z_{o}, z\right)$ can be exactly computed by exploiting the integration by part method.

For each $\left(z_{o}, z\right) \in\left[z_{\min }, z_{\max }\right] \times\left[z_{\min }, z_{\max }\right]:\left\{z_{o} \neq z\right\}$, if $\beta a>>1$, the integral above can be evaluated by resorting to an asymptotic approach. Since the choice of the asymptotic technique is related to the presence/absence of stationary points, it arises the problem of solving the equation

$$
\phi^{\prime}\left(x^{\prime}, z_{o}, z\right)=0 \quad \forall x^{\prime} \in[-a, a]
$$

where $\phi^{\prime}$ indicates the derivative of $\phi$ with respect to $x^{\prime}$. It is easy to show that if $\left|x_{o}\right|>a$ (or in other words if the observation domain is outside the region of the source), equation (7) does not admit solution any solution with respect to $x^{\prime}$ in the set $x^{\prime} \in[-a, a]$. Differently, if $\left|x_{o}\right|<a$ (or equivalently, if the observation domain is located in front of the source) then the point $x^{\prime}=x_{o}$ is a solution of such equation (see, once again, Fig. 1). For such reason, the cases $\left|x_{o}\right|>a$ and $\left|x_{o}\right|<a$ will be analyzed separately in sections IV and V, respectively.

\section{STUDY OF $\mathcal{T} \mathcal{T}_{W}^{\dagger}$ IN THE CASE $\left|x_{o}\right|>a$}

In this section, at first, the kernel of the operator $\mathcal{T} \mathcal{T}_{w}^{\dagger}$ in the case where $\left|x_{o}\right|>a$ is evaluated. Later, it is shown how to recast such operator in a form more similar to a convolution operator with a bandlimited kernel of sinc type.

As seen before, for $\left|x_{o}\right|>a$ no stationary points appear in the phase function; hence, the integral (6) can be asymptotically evaluated by taking into account only the contributions of the endpoints [37]. Consequently, if $\beta a>>1$ and $\left|x_{o}\right|>a$ then $\forall\left(z_{o}, z\right) \in\left[z_{\min }, z_{\max }\right] \times\left[z_{\min }, z_{\max }\right]:\left\{z_{o} \neq z\right\}$ the kernel of $\mathcal{T} \mathcal{T}_{w}^{\dagger}$ can be approximated by

$$
\begin{aligned}
& K\left(z_{o}, z\right) \approx \\
& -\left.\frac{z_{o} z}{j \beta a}\left(w\left(x^{\prime}, z\right) \frac{f\left(x^{\prime}, z_{o}, z\right)}{\phi^{\prime}\left(x^{\prime}, z_{o}, z\right)} e^{-j \beta a \phi\left(x^{\prime}, z_{o}, z\right)}\right)\right|_{x^{\prime}=-a} ^{x^{\prime}=a}
\end{aligned}
$$

From equation (8), it follows that

$$
\begin{aligned}
& K\left(z_{o}, z\right) \approx-\frac{z_{o} z}{j \beta a} e^{-j \frac{\beta a}{2}\left(\phi_{a}+\phi_{-a}\right)} . \\
& \quad\left(\frac{w_{a} f_{a}}{\phi_{a}{ }^{\prime}} e^{j \frac{\beta a}{2}\left(\phi_{-a}-\phi_{a}\right)}-\frac{w_{-a} f_{-a}}{\phi_{-a}{ }^{\prime}} e^{-j \frac{\beta a}{2}\left(\phi_{-a}-\phi_{a}\right)}\right)
\end{aligned}
$$

where the subscripts $-a$ or $a$ indicate that the correspondent function has been particularized in $x^{\prime}=-a$ or $x^{\prime}=a$.

As can be seen from (9), the operator $\mathcal{T} \mathcal{T}_{w}^{\dagger}$ is space-variant. In order to recast it in a form more similar to a convolution operator, let us introduce the following variables

$$
\begin{aligned}
& \zeta(z)=\frac{1}{2 a}\left(\sqrt{\left(x_{o}+a\right)^{2}+z^{2}}-\sqrt{\left(x_{o}-a\right)^{2}+z^{2}}\right) \\
& \gamma(z)=\frac{1}{2 a}\left(\sqrt{\left(x_{o}+a\right)^{2}+z^{2}}+\sqrt{\left(x_{o}-a\right)^{2}+z^{2}}\right)
\end{aligned}
$$

which represent the two elliptic coordinates. Thanks to the introduction of such variables, it results that $\frac{\beta a}{2}\left(\phi_{-a}-\phi_{a}\right)=$ $\beta a\left(\zeta_{o}-\zeta\right)$ and $\frac{\beta a}{2}\left(\phi_{-a}+\phi_{a}\right)=\beta a\left(\gamma\left(\zeta_{o}\right)-\gamma(\zeta)\right)$. Hence, by passing from the variables $\left(z_{o}, z\right)$ to the variables $\zeta_{o}=$ $\zeta\left(z_{o}\right)$ and $\zeta=\zeta(z)$, it is possible to recast (4) as below

$$
\mathcal{T} \mathcal{T}_{w}^{\dagger} E=\int_{\zeta\left(z_{\max }\right)}^{\zeta\left(z_{\min }\right)} K\left(\zeta_{o}, \zeta\right) E(\zeta) d \zeta
$$

where

$$
\begin{gathered}
K\left(\zeta_{o}, \zeta\right) \approx \frac{z\left(\zeta_{o}\right) z(\zeta)}{j \beta a} \frac{d z}{d \zeta} e^{-j \beta a\left[\gamma\left(\zeta_{o}\right)-\gamma(\zeta)\right]} \\
\left(\frac{w_{a}(\zeta) f_{a}\left(\zeta_{o}, \zeta\right)}{\phi_{a}{ }^{\prime}\left(\zeta_{o}, \zeta\right)} e^{j \beta a\left(\zeta_{o}-\zeta\right)}-\frac{w_{-a}(\zeta) f_{-a}\left(\zeta_{o}, \zeta\right)}{\phi_{-a}{ }^{\prime}\left(\zeta_{o}, \zeta\right)} e^{-j \beta a\left(\zeta_{o}-\zeta\right)}\right)
\end{gathered}
$$

The full expression of $z(\zeta)$ and its derivative $\frac{d z}{d \zeta}$ is reported in appendix A.

At this stage, the kernel has still a complicate structure. However, by approximating the amplitude factors as follows

$$
\begin{aligned}
\frac{z\left(\zeta_{o}\right) f_{a}\left(\zeta_{o}, \zeta\right)}{\phi_{a}^{\prime}\left(\zeta_{o}, \zeta\right)} & \approx \frac{a}{\left(x_{o}-a\right)\left(\zeta_{o}-\zeta\right) \frac{d z}{d \zeta}} \\
\frac{z\left(\zeta_{o}\right) f_{-a}\left(\zeta_{o}, \zeta\right)}{\phi_{-a}^{\prime}\left(\zeta_{o}, \zeta\right)} & \approx \frac{a}{\left(x_{o}+a\right)\left(\zeta_{o}-\zeta\right) \frac{d z}{d \zeta}}
\end{aligned}
$$

and by choosing

$$
w\left(x^{\prime}, \zeta\right)=\frac{\bar{w}\left(x^{\prime}\right)}{z(\zeta)}=\frac{\left(x_{o}-x^{\prime}\right)}{z(\zeta)},
$$

it can be rewritten in simple and nice form

$$
K\left(\zeta_{o}, \zeta\right) \approx \frac{2 \pi}{\beta} e^{j \beta a\left[\gamma(\zeta)-\gamma\left(\zeta_{o}\right)\right]} \frac{\sin \left(\beta a\left(\zeta_{o}-\zeta\right)\right)}{\pi\left(\zeta_{o}-\zeta\right)} .
$$

The derivation of the approximations (14) and (15) is shown in Appendix B.

Figures 2, 3 and 4 sketch respectively the kernel of $\mathcal{T} \mathcal{T}^{\dagger}$, $\mathcal{T} \mathcal{T}_{w}^{\dagger}$, and the sinc kernel (17). The diagrams are in $\mathrm{dB}$ and normalized with respect to its own maximum. As can be seen from such figures, the sinc kernel is almost equal to the actual kernel of $\mathcal{T} \mathcal{T}_{w}^{\dagger}$. Consequently, the eigenvalues of the 


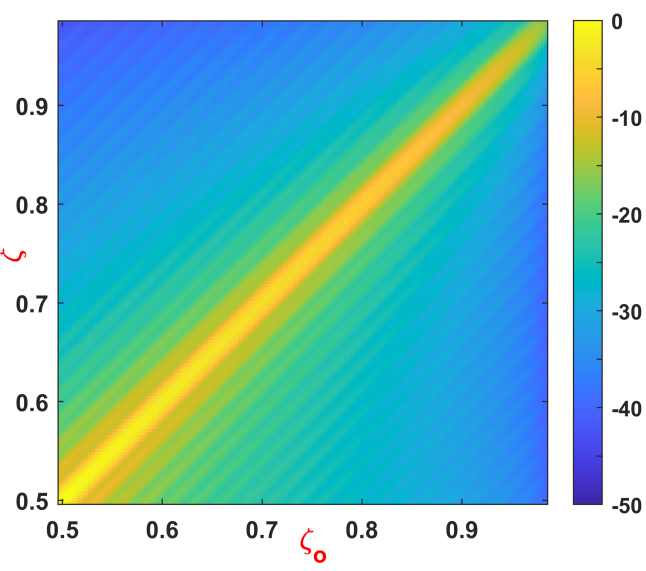

FIGURE 2. Kernel of $\mathcal{T} \mathcal{T}^{\dagger}$ in the variables $\left(\zeta, \zeta_{\mathrm{o}}\right)$ for $\mathbf{a}=\mathbf{2 0} \lambda$, $\mathrm{x}_{\mathrm{o}}=25 \lambda, \mathrm{z}_{\min }=2.5 \lambda\left(\zeta_{\max }=0.987\right), \mathrm{z}_{\max }=40 \lambda\left(\zeta_{\min }=0.497\right)$.

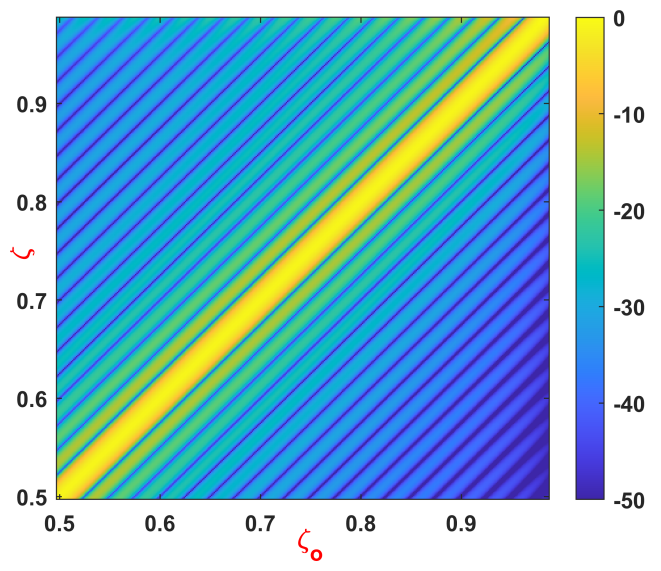

FIGURE 3. Kernel of $\mathcal{T} \mathcal{T}_{\mathrm{w}}^{\dagger}$ in the variables $\left(\zeta_{\mathrm{o}}, \zeta\right)$ for $\mathbf{a}=\mathbf{2 0} \lambda$, $\mathrm{x}_{\mathrm{o}}=25 \lambda, \mathrm{z}_{\min }=2.5 \lambda\left(\zeta_{\max }=0.987\right), \mathrm{z}_{\max }=40 \lambda$ $\left(\zeta_{\min }=0.497\right)$.

sinc kernel (17) approximate very well those of $\mathcal{T} \mathcal{T}_{w}^{\dagger}$. This is confirmed also by Fig. 5 in which the eigenvalues of $\mathcal{T T}^{\dagger}$, those of $\mathcal{T} \mathcal{T}_{w}^{\dagger}$, and those of the sinc kernel (17) are depicted. As can be seen from such figure, the eigenvalues of $\mathcal{T} \mathcal{T}_{w}^{\dagger}$ and those of the sinc kernel (17) have the same behavior, instead, the eigenvalues of $\mathcal{T} \mathcal{T}_{w}^{\dagger}$ exhibit a different behavior with respect to those of $\mathcal{T} \mathcal{T}^{\dagger}$. Despite this, the critical index beyond which the eigenvalues abrupt decay is the same for both the operators. This confirms that the use of the weighted adjoint does not change the number of relevant singular values but it modifies only the dynamics of the singular values by making them more flat.

From the above considerations, it follows that the number of relevant eigenvalues of $\mathcal{T} \mathcal{T}^{\dagger}$ (or in the words the NDF of the radiated field) can be evaluated by determining the number of relevant eigenvalues of $\mathcal{T} \mathcal{T}_{w}^{\dagger}$ which, in turn, exhibits the same eigenvalues of the integral operator with the sinc kernel (17).

Let us remark that despite the analysis developed above holds both for $x_{0}<-a$ and $x_{0}>a$, in the case where

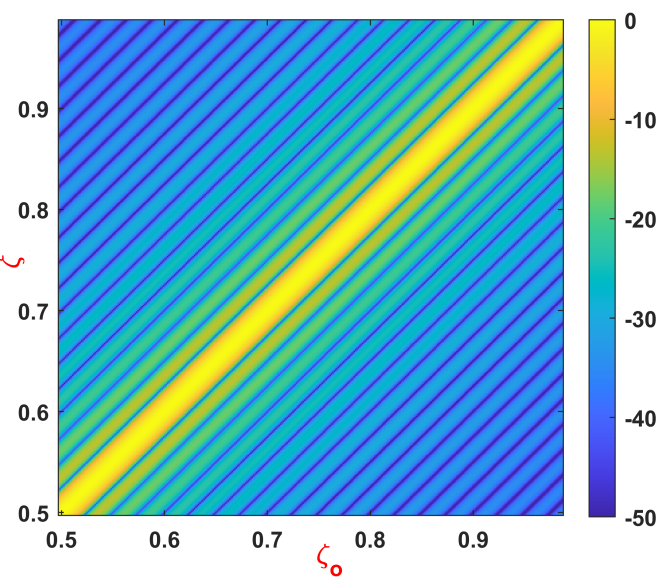

FIGURE 4. Sinc kernel given by (17) for $a=20 \lambda, x_{0}=25 \lambda$, $\mathbf{z}_{\min }=2.5 \lambda\left(\zeta_{\max }=0.987\right), \mathbf{z}_{\max }=40 \lambda\left(\zeta_{\min }=0.497\right)$.

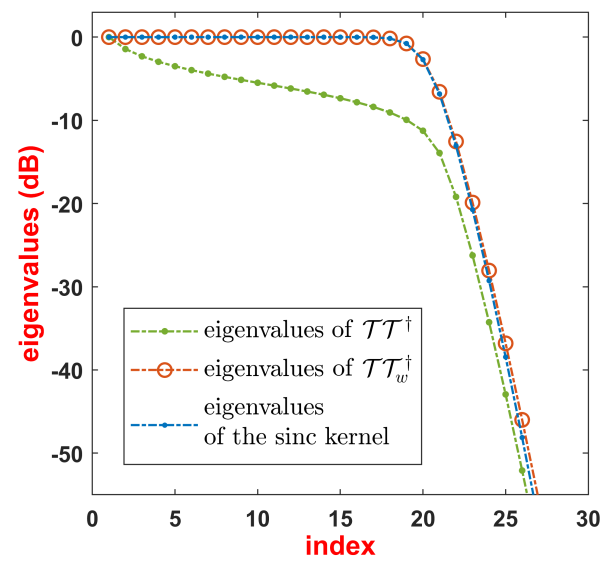

FIGURE 5. Eigenvalues of $\mathcal{T} \mathcal{T}^{\dagger}, \mathcal{T} \mathcal{T}_{\mathrm{w}}^{\dagger}$, and of the sinc kernel for $\mathrm{a}=20 \lambda, \mathrm{x}_{\mathrm{o}}=25 \lambda, \mathrm{z}_{\min }=2.5 \lambda\left(\zeta_{\max }=0.987\right), \mathrm{z}_{\max }=40 \lambda$ $\left(\zeta_{\min }=0.497\right)$.

$x_{o}<-a$ it may be convenient to define the variable $\zeta$ as the opposite of (10). In such a way, the kernel expression remains the same of (17) but the variable $\zeta$ attains positive values.

\section{STUDY OF $\mathcal{T} \mathcal{T}_{W}^{\dagger}$ IN THE CASE $\left|x_{o}\right| \leq a$}

In this section, with reference to the case where $\left|x_{o}\right| \leq a$, the kernel of $\mathcal{T} \mathcal{T}_{w}^{\dagger}$ is first evaluated, and after it is recast in a form suitable to be studied.

Differently from the case of section IV, if $\left|x_{o}\right|<a$ the asymptotic evaluation of the kernel contains not only the contributions of the endpoints $x^{\prime}=-a$ and $x^{\prime}=a$ but also the contribution given by the stationary point $x^{\prime}=x_{o}$ [37]. Hence, if $\beta a>>1$ and $\left|x_{o}\right|<a$ an asymptotic evaluation of the integral (6) which holds true $\forall\left(z_{o}, z\right) \in$ $\left[z_{\min }, z_{\max }\right] \times\left[z_{\min }, z_{\max }\right]:\left\{z_{o} \neq z\right\}$ is given by

$$
\begin{aligned}
K\left(z_{o}, z\right) \approx z_{o} z & \left(\frac{w_{a} f_{a}}{-j \beta a \phi_{a}^{\prime}} e^{-j \beta a \phi_{a}}-\frac{w_{-a} f_{-a}}{-j \beta a \phi_{-a}{ }^{\prime}} e^{-j \beta a \phi_{-a}}\right. \\
& \left.+\sqrt{\frac{2 \pi}{\beta a}} \frac{w_{x_{o}} f_{x_{o}}}{\sqrt{\left|\phi_{x_{o}}^{\prime \prime}\right|}} e^{-j \beta a \phi_{x_{o}}} e^{j \frac{\pi}{4} \operatorname{sign}\left(-\phi_{x_{o}}^{\prime \prime}\right)}\right)
\end{aligned}
$$


where

- $\phi^{\prime \prime}$ indicates the second partial derivative of $\phi$ with respect to the variable $x^{\prime}$,

- the subscripts $-a, a, x_{o}$ denote respectively that the corresponding function has been particularized for $x^{\prime}=$ $-a, x^{\prime}=a$ or $x^{\prime}=x_{o}$.

To further proceed in our analysis, let us restrict the attention to the case $0<x_{o}<a$. In such circumstance, it is convenient to recast the previous expression of $K\left(z_{o}, z\right)$ in the form

$K\left(z_{o}, z\right) \approx z_{o} z e^{-j \frac{\beta a}{2}\left(\phi_{-a}+\phi_{x_{o}}\right)}\left(\frac{w_{-a} f_{-a}}{j \beta a \phi_{-a}^{\prime}} e^{-j \frac{\beta a}{2}\left(\phi_{-a}-\phi_{x_{o}}\right)}\right.$
$\left.+\sqrt{\frac{2 \pi}{\beta a}} \frac{w_{x_{o}} f_{x_{o}}}{\sqrt{\left|\phi_{x_{o}}^{\prime \prime}\right|}} e^{j \frac{\beta a}{2}\left(\phi_{-a}-\phi_{x_{o}}\right)} e^{j \frac{\pi}{4} \operatorname{sign}\left(z_{o}-z\right)}\right)-\Delta K\left(z_{o}, z\right)$

where

$$
\Delta K_{a}\left(z_{o}, z\right)=\frac{z_{o} z}{j \beta a} \frac{w_{a}(z) f_{a}\left(z_{o}, z\right)}{\phi_{a}{ }^{\prime}\left(z_{o}, z\right)} e^{-j \beta a \phi_{a}\left(z_{o}, z\right)} .
$$

With the aim to obtain a kernel whose eigenvalues can be computed in closed form, let us introduce the variables

$$
\begin{aligned}
& \zeta(z)=\frac{1}{a+x_{o}}\left(\sqrt{\left(x_{o}+a\right)^{2}+z^{2}}-|z|\right) \\
& \gamma(z)=\frac{1}{a+x_{o}}\left(\sqrt{\left(x_{o}+a\right)^{2}+z^{2}}+|z|\right)
\end{aligned}
$$

The latter allow writing that $a\left(\phi_{-a}-\phi_{x_{o}}\right)=\left(a+x_{o}\right)\left(\zeta_{o}-\zeta\right)$ and $a\left(\phi_{-a}\left(\zeta_{o}, \zeta\right)+\phi_{x_{o}}\left(\zeta_{o}, \zeta\right)\right)=\left(a+x_{o}\right)\left(\gamma\left(\zeta_{o}\right)-\gamma(\zeta)\right)$. Hence, by expressing (3) in terms of the variables $\zeta_{o}=\zeta\left(z_{o}\right)$ and $\zeta=\zeta(z)$, it results that

$$
\mathcal{T} \mathcal{T}_{w}^{\dagger} E=\int_{\zeta\left(z_{\max }\right)}^{\zeta\left(z_{\min }\right)} K\left(\zeta_{o}, \zeta\right) E(\zeta) d \zeta
$$

where

$$
\begin{aligned}
& K\left(\zeta_{o}, \zeta\right) \approx-z\left(\zeta_{o}\right) z(\zeta) \frac{d z}{d \zeta} e^{-j \frac{\beta\left(a+x_{o}\right)}{2}\left(\gamma\left(\zeta_{o}\right)-\gamma(\zeta)\right)} \\
& \left(w_{-a}(\zeta) \frac{f_{-a}\left(\zeta_{o}, \zeta\right)}{j \beta a \phi_{-a}^{\prime}\left(\zeta_{o}, \zeta\right)} e^{-j \frac{\beta\left(a+x_{o}\right)}{2}\left(\zeta_{o}-\zeta\right)}+\sqrt{\frac{2 \pi}{\beta a}} w_{x_{o}}(\zeta)\right. \\
& \left.\frac{f_{x_{o}}\left(\zeta_{o}, \zeta\right)}{\sqrt{\left|\phi_{x_{o}}^{\prime \prime}\left(\zeta_{o}, \zeta\right)\right|}} e^{j \frac{\beta\left(a+x_{o}\right)}{2}\left(\zeta_{o}-\zeta\right)} e^{-j \frac{\pi}{4} \operatorname{sign}\left(\zeta_{o}-\zeta\right)}\right)+\Delta K_{a}\left(\zeta_{o}, \zeta\right)
\end{aligned}
$$

with

$$
\Delta K_{a}\left(\zeta_{o}, \zeta\right)=\frac{z\left(\zeta_{o}\right) z(\zeta)}{j \beta a} \frac{w_{a}(\zeta) f_{a}\left(\zeta_{o}, \zeta\right)}{\phi_{a}{ }^{\prime}\left(\zeta_{o}, \zeta\right)} \frac{d z}{d \zeta} e^{-j \beta a \phi_{a}\left(\zeta_{o}, \zeta\right)}
$$

The full expression of $z(\zeta)$ and $\frac{d z}{d \zeta}$ is shown in Appendix C. The first term of $K\left(\zeta_{o}, \zeta\right)$ can be expressed in terms of a sinc function depending on the difference $\left(\zeta_{o}-\zeta\right)$. In order to achieve this aim, first let us approximate the amplitude term $z\left(\zeta_{o}\right) f_{-a}\left(\zeta_{o}, \zeta\right) / \phi_{-a}{ }^{\prime}\left(\zeta_{o}, \zeta\right)$ as in (15), and $f_{x_{o}}\left(\zeta_{o}, \zeta\right) / \sqrt{\phi_{x_{o}}^{\prime \prime}\left(\zeta_{o}, \zeta\right)}$ as below

$$
\frac{f_{x_{o}}\left(\zeta_{o}, \zeta\right)}{\sqrt{\left|\phi_{x_{o}}^{\prime \prime}\left(\zeta_{o}, \zeta\right)\right|}} \approx \frac{\sqrt{a}}{2 z(\zeta) z\left(\zeta_{o}\right)} \frac{\operatorname{sign}\left(\zeta_{o}-\zeta\right)}{\sqrt{\left|\frac{d z}{d \zeta}\right|}\left(\zeta_{o}-\zeta\right)}
$$

(see Appendix D for the derivation of approximation (26)). Later, let us choose the following weight function

$$
w\left(x^{\prime}, \zeta\right)=\frac{\bar{w}\left(x^{\prime}\right)}{z(\zeta)} \approx \frac{\frac{\bar{w}_{-a}-\bar{w}_{x_{o}}}{-a-x_{o}}\left(x^{\prime}-x_{o}\right)+\bar{w}_{x_{o}}}{z(\zeta)}
$$

where

$$
\bar{w}_{-a}=\frac{\pi\left(a+x_{o}\right)}{\lambda} \quad, \quad \bar{w}_{x_{o}}=\sqrt{\frac{a+x_{o}}{2 \lambda}} .
$$

By taking into account of equations (15) (26) (28), and by performing the following approximation

$$
\frac{1}{z(\zeta)} \sqrt{\left|\frac{d z}{d \zeta}\right|}=\frac{\sqrt{2}}{\sqrt{a+x_{o}}} \frac{\sqrt{1+\zeta^{2}}}{1-\zeta^{2}} \approx \sqrt{\frac{2}{a+x_{o}}}
$$

the kernel expressed in (24) can be approximated as follows

$$
\begin{aligned}
& K\left(\zeta_{o}, \zeta\right) \approx \pi e^{-j \frac{\beta\left(a+x_{o}\right)}{2}\left(\gamma\left(\zeta_{o}\right)-\gamma(\zeta)\right)} e^{j \frac{\pi}{8} \operatorname{sign}\left(\zeta_{o}-\zeta\right)} \\
& \frac{\sin \left(\frac{\beta\left(a+x_{o}\right)}{2}\left(\zeta_{o}-\zeta\right)+\frac{\pi}{8} \operatorname{sign}\left(\zeta_{o}-\zeta\right)\right)}{\pi\left(\zeta_{o}-\zeta\right)}+\Delta K_{a}\left(\zeta_{o}, \zeta\right)
\end{aligned}
$$

Since equation (18) does not work for $z_{o}=z$, equation (30) is not valid for $\zeta_{o}=\zeta$. An expression of $K\left(\zeta_{o}, \zeta\right)$ which well approximates the behavior of the actual kernel $\forall\left(\zeta_{o}, \zeta\right) \in\left[\zeta_{\min }, \zeta_{\max }\right] \times\left[\zeta_{\min }, \zeta_{\max }\right]$ is given by

$$
\begin{aligned}
K\left(\zeta_{o}, \zeta\right) \approx & \pi e^{-j \frac{\beta\left(a+x_{o}\right)}{2}\left(\gamma\left(\zeta_{o}\right)-\gamma(\zeta)\right)} \frac{\sin \left(\frac{\beta\left(a+x_{o}\right)}{2}\left(\zeta_{o}-\zeta\right)\right)}{\pi\left(\zeta_{o}-\zeta\right)} \\
& +\Delta K_{a}\left(\zeta_{o}, \zeta\right)
\end{aligned}
$$

Hence, for $0<x_{o}<a$ the kernel of $\mathcal{T} \mathcal{T}_{w}^{\dagger}$ is made up by two terms. The first one is proportional to a sinc kernel of difference type, instead, the second term $\Delta K_{a}$ can be regarded as an additive error.

Figures 6, 7 and 8 sketch respectively the actual kernel of $\mathcal{T}^{\dagger}, \mathcal{T} \mathcal{T}_{w}^{\dagger}$, and the first term of (31). The diagrams are in $\mathrm{dB}$ and normalized with respect to its own maximum. As it can be seen from such figures, the kernel of $\mathcal{T} \mathcal{T}^{\dagger}$ exhibits a different decay with respect to the others. Instead, the kernel of $\mathcal{T} \mathcal{T}_{w}^{\dagger}$ and the sinc term in (31) strongly differ in the region outside the main lobe whereas they are similar in the region of the main lobe (in particular way for small and medium values of $\zeta$ and $\zeta_{o}$ ). In this last case, the differences between the two diagrams are due essentially to the approximation made in (29), and to the fact that we are neglecting the additive term $\Delta K$.

Despite the differences among Figs. 6, 7 and 8, the number of significant eigenvalues is essentially the same in all the three cases. This aspect is well shown in Fig. 9 which sketches the eigenvalues of $\mathcal{T} \mathcal{T}^{\dagger}, \mathcal{T} \mathcal{T}_{w}^{\dagger}$ and those of the integral operator with the sinc kernel. As it can be seen from Fig. 9, the dynamics of the eigenvalues changes in each case, instead, the position of the knee in the eigenvalues curve is always the same. Accordingly, also for $0<x_{o}<a$ the number of relevant eigenvalues of $\mathcal{T T}^{\dagger}$ can be computed 


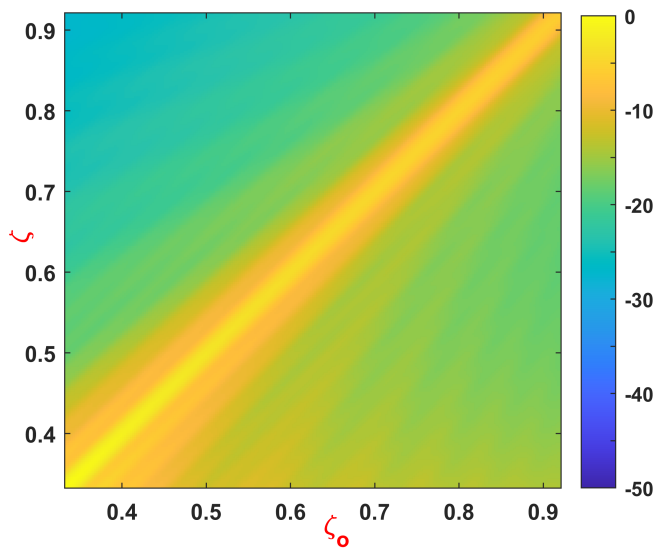

FIGURE 6. Kernel of $\mathcal{T} \mathcal{T}^{\dagger}$ in the variables $\left(\zeta_{\mathrm{o}}, \zeta\right)$ for $\mathbf{a}=\mathbf{2 0} \lambda$, $\mathrm{x}_{\mathrm{o}}=10 \lambda, \mathrm{z}_{\min }=2.5 \lambda,\left(\zeta_{\max }=0.920\right), \mathrm{z}_{\max }=40 \lambda$ $\left(\zeta_{\min }=0.333\right)$.

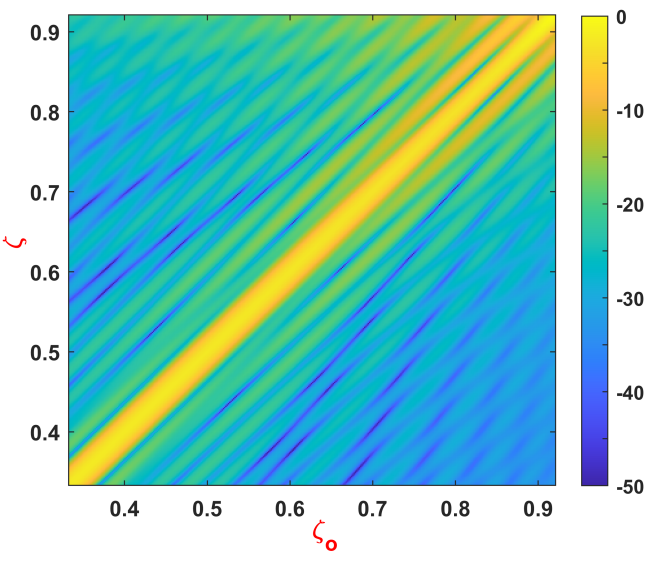

FIGURE 7. Kernel of $\mathcal{T} \mathcal{T}_{\mathbf{w}}^{\dagger}$ in the variables $\left(\zeta_{\mathrm{o}}, \zeta\right)$ for $\mathbf{a}=\mathbf{2 0} \lambda$, $\mathrm{x}_{\mathrm{o}}=10 \lambda, \mathrm{z}_{\min }=2.5 \lambda\left(\zeta_{\max }=0.920\right), \mathrm{z}_{\max }=40 \lambda$ $\left(\zeta_{\min }=0.333\right)$.

by referring to the integral operator with the sinc kernel corresponding to the first term of (31).

It is worth noting that the study developed for $0<x_{o}<a$ can be repeated in a dual way for $-a<x_{o}<0$. In such a case, the terms of (18) that allow approximating the main lobe of the kernel of $T T_{w}^{\dagger}$ are those due to the endpoint $x^{\prime}=a$ and to the stationary point $x^{\prime}=0$. Hence, after the introduction of the variables

$$
\begin{aligned}
& \zeta(z)=\frac{1}{a-x_{o}}\left(\sqrt{\left(x_{o}-a\right)^{2}+z^{2}}-|z|\right) \\
& \gamma(z)=\frac{1}{a-x_{o}}\left(\sqrt{\left(x_{o}-a\right)^{2}+z^{2}}+|z|\right),
\end{aligned}
$$

it is possible to approximate the kernel of $T T_{w}^{\dagger}$ in the case where $-a<x_{o}<0$ as below

$$
\begin{aligned}
K\left(\zeta_{o}, \zeta\right) \approx & \pi e^{-j \frac{\beta\left(a-x_{o}\right)}{2}\left(\gamma\left(\zeta_{o}\right)-\gamma(\zeta)\right)} \frac{\sin \left(\frac{\beta\left(a-x_{o}\right)}{2}\left(\zeta_{o}-\zeta\right)\right)}{\pi\left(\zeta_{o}-\zeta\right)} \\
& +\Delta K_{-a}\left(\zeta_{o}, \zeta\right)
\end{aligned}
$$

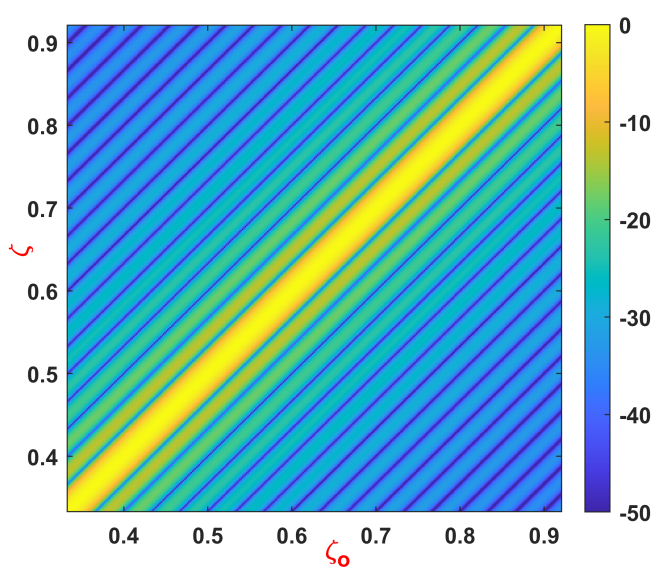

FIGURE 8. Sinc kernel corresponding to the first term of (31) for $\mathrm{a}=20 \lambda, \mathrm{x}_{\mathrm{o}}=10 \lambda, \mathrm{z}_{\min }=2.5 \lambda\left(\zeta_{\max }=0.920\right), \mathrm{z}_{\max }=40 \lambda$ $\left(\zeta_{\min }=0.333\right)$.

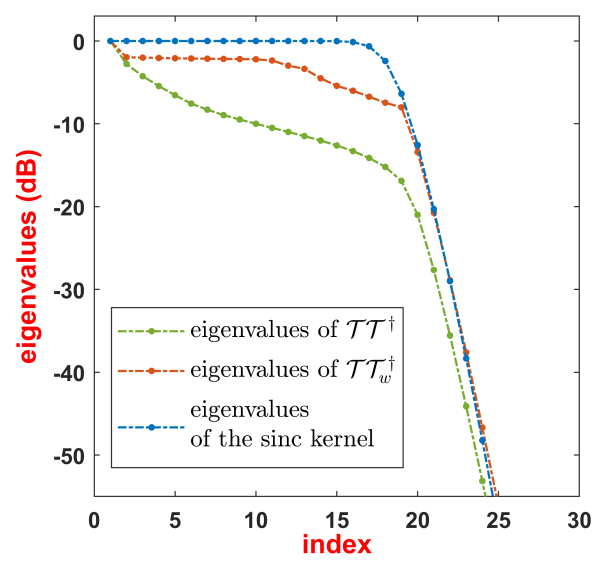

FIGURE 9. Eigenvalues of $\mathcal{T} \mathcal{T}^{\dagger}, \mathcal{T} \mathcal{T}_{\mathrm{w}}^{\dagger}$, and of the sinc kernel for $\mathrm{a}=20 \lambda, \mathrm{x}_{\mathrm{o}}=10 \lambda, \mathrm{z}_{\min }=2.5 \lambda\left(\zeta_{\max }=0.920\right), \mathrm{z}_{\max }=40 \lambda$ $\left(\zeta_{\min }=0.333\right)$.

As regards the eigenvalues behavior, the discussion already made for the case $0<x_{o}<a$ works also for $-a<x_{o}<0$.

At this point, let us tackle the cases where $x_{o}=0$ and $x_{o}= \pm a$. In such circumstance, the asymptotic evaluation of the kernel of $\mathcal{T} \mathcal{T}_{w}^{\dagger}$ can be expressed only by two complex exponential functions; accordingly, it can be recast as a purely sinc function.

\section{A. THE CASE $\mathrm{x}_{\mathrm{o}}=0$}

In this section, a closed-form expression of the kernel of $\mathcal{T} \mathcal{T}_{w}^{\dagger}$ in the case where $x_{o}=0$ is provided. In such a case, the asymptotic evaluation of $K\left(z_{o}, z\right)$ given by equation (18) works $\forall\left(z_{o}, z\right) \in\left[z_{\min }, z_{\max }\right] \times\left[z_{\min }, z_{\max }\right]:\left\{z_{o} \neq z\right\}$. Furthermore, since $x_{o}=0$, it results also that

$$
\phi_{a}\left(z_{o}, z\right)=\phi_{-a}\left(z_{o}, z\right), \frac{f_{a}\left(z_{o}, z\right)}{\phi_{a}^{\prime}\left(z_{o}, z\right)}=-\frac{f_{-a}\left(z_{o}, z\right)}{\phi_{-a}^{\prime}\left(z_{o}, z\right)}
$$




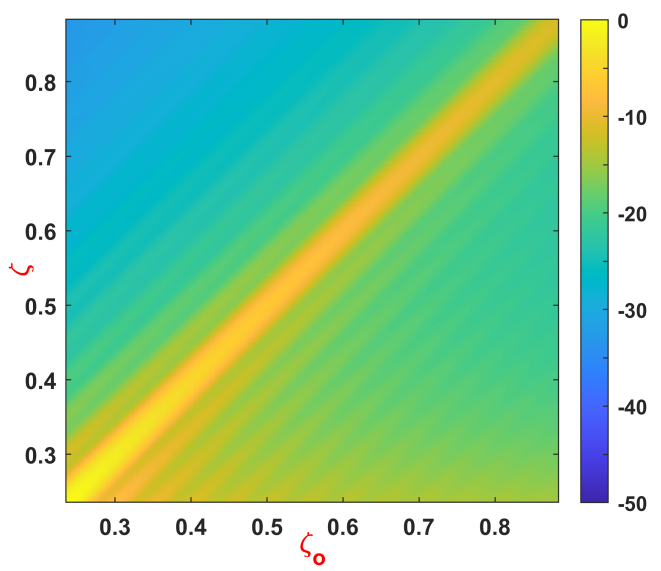

FIGURE 10. Kernel of $\mathcal{T} \mathcal{T}^{\dagger}$ in the variables $\left(\zeta_{\mathrm{o}}, \zeta\right)$ for $\mathbf{a}=\mathbf{2 0} \lambda$, $\mathrm{x}_{\mathrm{o}}=0 \lambda, \mathrm{z}_{\min }=2.5 \lambda\left(\zeta_{\max }=0.883\right), \mathrm{z}_{\max }=40 \lambda\left(\zeta_{\min }=0.236\right)$.

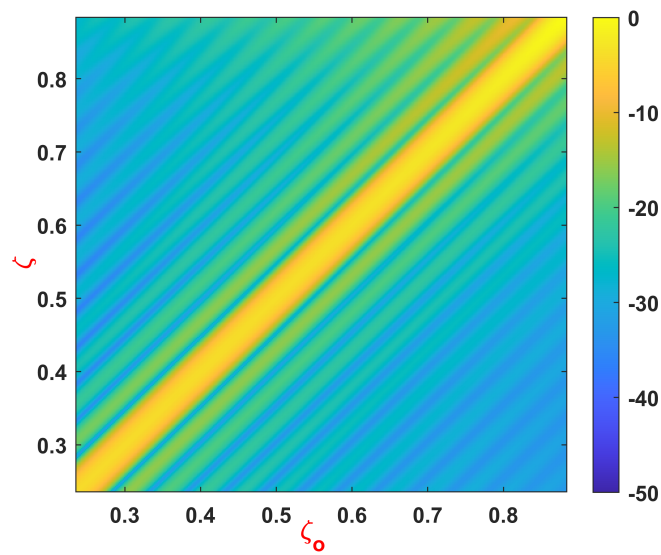

FIGURE 11. Kernel of $\mathcal{T} \mathcal{T}_{\mathrm{w}}^{\dagger}$ in the variables $\left(\zeta_{\mathrm{o}}, \zeta\right)$ for $\mathbf{a}=\mathbf{2 0} \lambda$ $\mathrm{x}_{\mathrm{o}}=0 \lambda, \mathrm{z}_{\min }=2.5 \lambda\left(\zeta_{\max }=0.883\right), \mathrm{z}_{\max }=40 \lambda\left(\zeta_{\min }=0.236\right)$.

Consequently, if the weight function $w\left(x^{\prime}, z\right)$ is such that $w_{a}(z)=w_{-a}(z)$, the kernel (18) can be rewritten as

$$
\begin{aligned}
K\left(z_{o}, z\right) \approx & z_{o} z\left(\frac{2 w_{-a} f_{-a}}{j \beta a \phi_{-a}} e^{-j \beta a \phi_{-a}}+\right. \\
& \left.+\sqrt{\frac{2 \pi}{\beta a}} \frac{w_{0} f_{0}}{\sqrt{\left|\phi_{0}^{\prime \prime}\right|}} e^{-j \beta a \phi_{0}} e^{j \frac{\pi}{4} \operatorname{sign}\left(-\phi_{0}^{\prime \prime}\right)}\right)
\end{aligned}
$$

where the subscript 0 denotes the the correspondent function has been particularized for $x^{\prime}=x_{o}=0$.

The use of the variables $\zeta(z)$ and $\gamma(z)$ defined in (21) and (22), and the following choice of the weight function

$$
w\left(x^{\prime}, \zeta\right)=\frac{\bar{w}\left(x^{\prime}\right)}{z(\zeta)}=\frac{\frac{\bar{w}_{-a}-\bar{w}_{0}}{-a}\left|x^{\prime}\right|+\bar{w}_{0}}{z(\zeta)}
$$

where $\bar{w}_{-a}=\frac{\pi a}{\lambda}, \bar{w}_{0}=\sqrt{\frac{2 a}{\lambda}}$ allow recasting the kernel of $\mathcal{T} \mathcal{T}_{w}^{\dagger}$ as below

$$
K\left(\zeta_{o}, \zeta\right) \approx 2 \pi e^{-j \frac{\beta a}{2}\left(\gamma\left(\zeta_{o}\right)-\gamma(\zeta)\right)} \frac{\sin \left(\frac{\beta a}{2}\left(\zeta_{o}-\zeta\right)\right)}{\pi\left(\zeta_{o}-\zeta\right)} .
$$

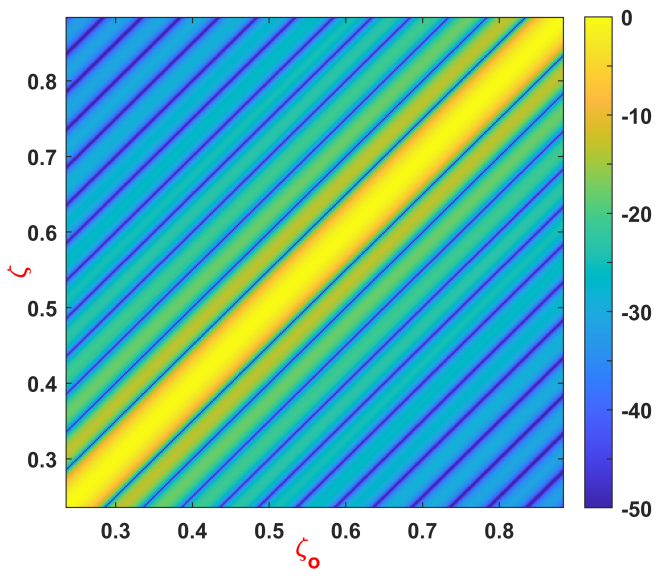

FIGURE 12. Sinc kernel given by (38) for $\mathrm{a}=\mathbf{2 0} \lambda, \mathrm{x}_{\mathrm{o}}=0 \lambda$, $\mathrm{z}_{\min }=2.5 \lambda\left(\zeta_{\max }=0.883\right), \mathrm{z}_{\max }=40 \lambda\left(\zeta_{\min }=0.236\right)$.

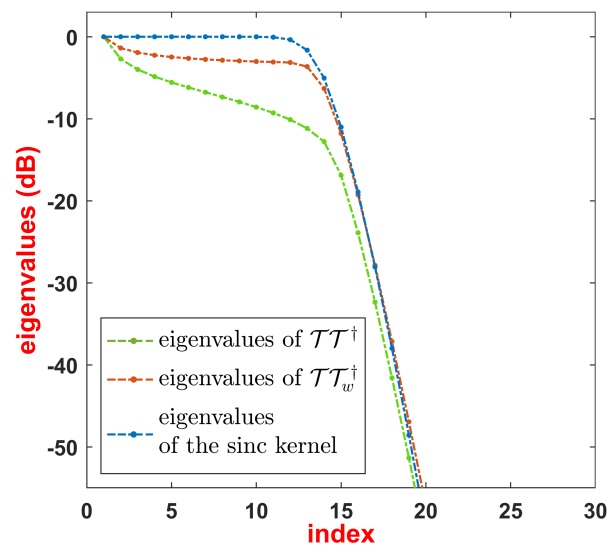

FIGURE 13. Eigenvalues of $\mathcal{T} \mathcal{T}^{\dagger}, \mathcal{T} \mathcal{T}_{\mathrm{w}}^{\dagger}$, and of the sinc kernel for $\mathrm{a}=20 \lambda, \mathrm{x}_{\mathrm{o}}=0 \lambda, \mathrm{z}_{\min }=2.5 \lambda\left(\zeta_{\max }=0.883\right), \mathrm{z}_{\max }=40 \lambda$ $\left(\zeta_{\min }=0.236\right)$.

In Figs. 10, 11 and 12 the kernel of $\mathcal{T} \mathcal{T}^{\dagger}, \mathcal{T} \mathcal{T}_{w}^{\dagger}$, and the sinc kernel (38) are sketched in $\mathrm{dB}$ and normalized with respect to its own maximum.

Note that when $x_{o}=0$, the sinc kernel expressed by (38) approximates well the actual kernel of $\mathcal{T} \mathcal{T}_{w}^{\dagger}$. The only difference between the two diagrams concerns the value of the two kernels along the lines given by the equation $\zeta_{o}-\zeta=n \frac{2 \pi}{\beta a}$. Along such lines, the sinc kernel is exactly zero, instead, the actual kernel of $\mathcal{T} \mathcal{T}_{w}^{\dagger}$ assumes a value that is $-35 d B$ with respect to its maximum.

In Fig. 13 the eigenvalues of $\mathcal{T} \mathcal{T}^{\dagger}, \mathcal{T} \mathcal{T}_{w}^{\dagger}$ and those of the integral operator with the sinc kernel (38) are depicted. As it can be seen from the figure, also in this case the number of relevant eigenvalues is the same in all the cases. Consequently, also for $x_{o}=0$ the number of relevant eigenvalues of $\mathcal{T T}^{\dagger}$ can be computed by referring to integral operator with the sinc kernel (38). 


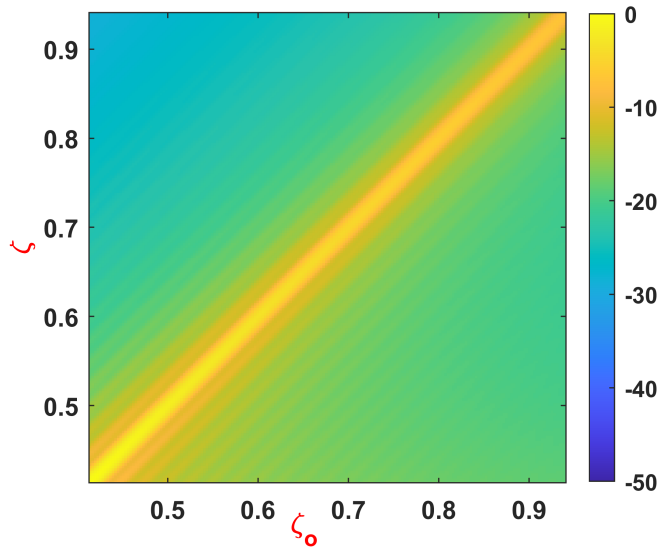

FIGURE 14. Kernel of $\mathcal{T} \mathcal{T}^{\dagger}$ in the variables $\left(\zeta_{0}, \zeta\right)$ for $\mathbf{a}=\mathbf{2 0} \lambda$, $\mathrm{x}_{\mathrm{o}}=20 \lambda, \mathrm{z}_{\min }=2.5 \lambda\left(\zeta_{\max }=0.939\right), \mathrm{z}_{\max }=40 \lambda$ $\left(\zeta_{\min }=0.415\right)$

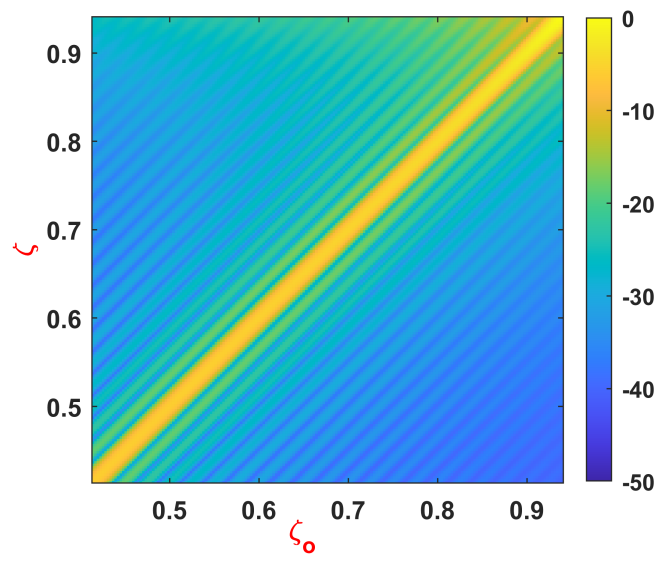

FIGURE 15. Kernel of $\mathcal{T} \mathcal{T}_{\mathrm{w}}^{\dagger}$ in the variables $\left(\zeta_{\mathrm{o}}, \zeta\right)$ for $\mathbf{a}=\mathbf{2 0} \lambda$, $\mathrm{x}_{\mathrm{o}}=20 \lambda, \mathrm{z}_{\min }=2.5 \lambda\left(\zeta_{\max }=0.939\right), \mathrm{z}_{\max }=40 \lambda$ $\left(\zeta_{\min }=0.415\right)$.

\section{B. THE CASES $\mathrm{x}_{\mathrm{o}}=\mathrm{a} A N D \mathrm{x}_{\mathrm{o}}=-\mathrm{a}$}

In this section, the operator $\mathcal{T} \mathcal{T}_{w}^{\dagger}$ is studied in the case where $x_{o}=a$ and $x_{o}=-a$.

For $x_{o}=a$, the point $x^{\prime}=a$ is at the same time an endpoint and a stationary point; hence, the asymptotic evaluation of the $K\left(z_{o}, z\right)$ is given by

$$
\begin{aligned}
K\left(z_{o}, z\right) \approx & z_{o} z\left(\frac{w_{-a} f_{-a}}{j \beta a \phi_{-a}^{\prime}} e^{-j \beta a \phi_{-a}}+\right. \\
& \left.+\frac{1}{2} \sqrt{\frac{2 \pi}{\beta a}} \frac{w_{a} f_{a}}{\sqrt{\left|\phi_{a}^{\prime \prime}\right|}} e^{-j \beta a \phi_{a}} e^{j \frac{\pi}{4} \operatorname{sign}\left(-\phi_{a}^{\prime \prime}\right)}\right)
\end{aligned}
$$

Keeping in mind the definition of $\zeta(z)$ and $\gamma(z)$ provided respectively in (21) and (22), and choosing the weight function as below

$$
w\left(x^{\prime}, \zeta\right)=\frac{\bar{w}\left(x^{\prime}\right)}{z(\zeta)}=\frac{\frac{\bar{w}_{-a}-\bar{w}_{a}}{-a-a} x^{\prime}+\bar{w}_{a}}{z(\zeta)}
$$

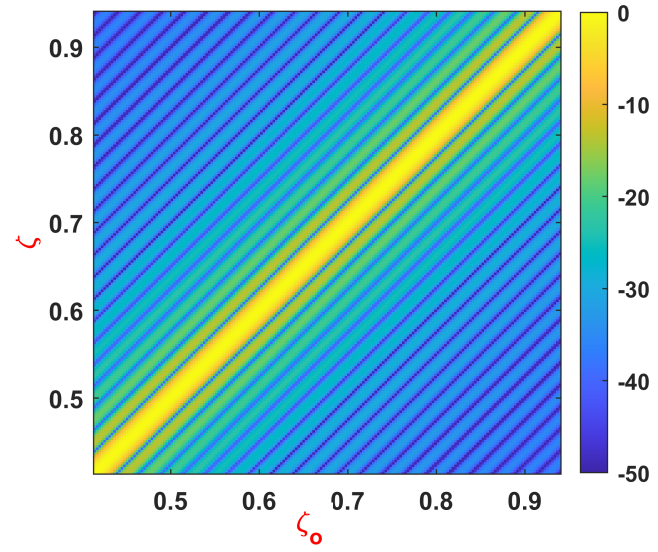

FIGURE 16. Sinc kernel given by equation (41) for $a=20 \lambda, x_{0}=20 \lambda$, $\mathrm{z}_{\min }=2.5 \lambda\left(\zeta_{\max }=0.939\right), \mathrm{z}_{\max }=40 \lambda\left(\zeta_{\min }=0.415\right)$.

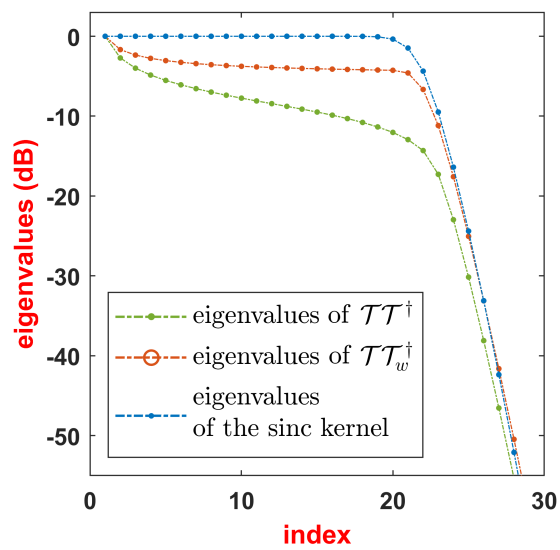

FIGURE 17. Eigenvalues of $\mathcal{T} \mathcal{T}^{\dagger}, \mathcal{T} \mathcal{T}_{\mathrm{w}}^{\dagger}$, and of the sinc kernel for $\mathrm{a}=20 \lambda, \mathrm{x}_{\mathrm{o}}=20 \lambda, \mathrm{z}_{\min }=2.5 \lambda\left(\zeta_{\max }=0.939\right), \mathrm{z}_{\max }=40 \lambda$ $\left(\zeta_{\min }=0.415\right)$.

with $\bar{w}_{-a}=\frac{2 \pi a}{\lambda}$ and $\bar{w}_{a}=\sqrt{\frac{4 a}{\lambda}}$, it is possible to approximate the kernel of $\mathcal{T} \mathcal{T}_{w}^{\dagger}$ by the following expression

$$
K\left(\zeta_{o}, \zeta\right) \approx \pi e^{-j \beta a\left(\gamma\left(\zeta_{o}\right)-\gamma(\zeta)\right)} \frac{\sin \left(\beta a\left(\zeta_{o}-\zeta\right)\right)}{\pi\left(\zeta_{o}-\zeta\right)} .
$$

In Figs. 14, 15 and 16 the kernel of $\mathcal{T} \mathcal{T}^{\dagger}, \mathcal{T} \mathcal{T}_{w}^{\dagger}$, and the sinc kernel are sketched in $d B$. Instead, in Fig. 17 the eigenvalues of $\mathcal{T} \mathcal{T}^{\dagger}, \mathcal{T} \mathcal{T}_{w}^{\dagger}$ and those of the sinc kernel (41) are shown. All the considerations already made in the case $x_{o}=0$ hold true also for $x_{o}=a$.

The study of the operator $\mathcal{T} \mathcal{T}_{w}^{\dagger}$ for $x_{o}=-a$ can be tackled as the case $x_{o}=a$. For $x_{o}=-a$, the variables $\zeta$ and $\gamma$ have the expression given by (32) and (33), respectively. Despite the definition of the variables $\zeta$ and $\gamma$ is different by the case $x_{o}=a$, the formal expression of $K\left(\zeta_{o}, \zeta\right)$ will be once again that provided by (41).

\section{NDF OF THE RADIATED FIELD}

In the previous sections, it has been shown that the eigenvalues of $\mathcal{T} \mathcal{T}^{\dagger}$ can exhibit a different dynamics from those of the correspondent integral operator with a sinc kernel; however, 


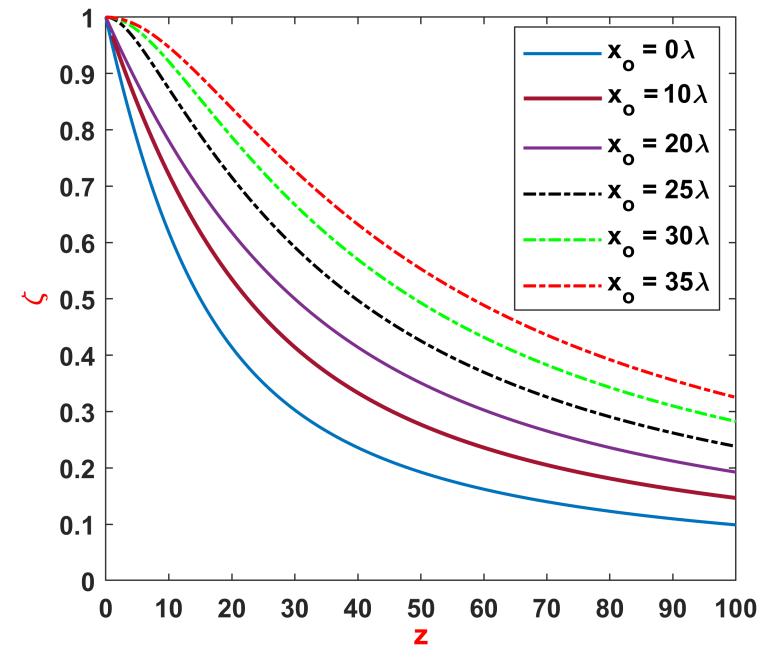

FIGURE 18. Behavior of the transformation $\zeta(\mathbf{z})$ for different values of $\mathrm{x}_{\mathrm{o}}$ when $\mathrm{a}=20 \lambda$.

in each case, the position of the knee in the eigenvalues diagrams occurs at the same index. For such reason, the number of degrees of freedom (that corresponds to the number of eigenvalues before the knee) can be evaluated by referring to the integral operator

$$
\int_{\hat{\zeta}\left(z_{\max }\right)}^{\hat{\zeta}\left(z_{\min }\right)} e^{j W\left(\hat{\gamma}(\zeta)-\hat{\gamma}\left(\zeta_{o}\right)\right)} \frac{\sin \left(W\left(\hat{\zeta}_{o}-\hat{\zeta}\right)\right)}{\pi\left(\hat{\zeta}_{o}-\hat{\zeta}\right)}(.) d \zeta
$$

where

$$
W=\left\{\begin{array}{lll}
\beta a & \text { for } & \left|x_{o}\right| \geq a \\
\beta \frac{a+\left|x_{o}\right|}{2} & \text { for } & \left|x_{o}\right| \leq a
\end{array}\right.
$$

By exploiting the Slepian-Pollak results in [34], it follows that the number of degrees of freedom is given by

$$
N D F=\frac{W}{\pi}\left(\hat{\zeta}\left(z_{\min }\right)-\hat{\zeta}\left(z_{\max }\right)\right)
$$

where $\hat{\zeta}(z)$ is defined as

$$
\hat{\zeta}(z)=\left\{\begin{array}{lll}
\frac{\sqrt{\left(x_{o}+a\right)^{2}+z^{2}}-\sqrt{\left(x_{o}-a\right)^{2}+z^{2}}}{2 a} & \text { for } x_{o}>a \\
\frac{\sqrt{\left(x_{o}+a\right)^{2}+z^{2}}-|z|}{a+x_{o}} & \text { for } \quad 0 \leq x_{o} \leq a \\
\frac{\sqrt{\left(x_{o}-a\right)^{2}+z^{2}}-|z|}{a-x_{o}} & \text { for } \quad-a \leq x_{o} \leq 0 \\
\frac{\sqrt{\left(x_{o}-a\right)^{2}+z^{2}}-\sqrt{\left(x_{o}+a\right)^{2}+z^{2}}}{2 a} & \text { for } x_{o}<-a
\end{array}\right.
$$

In Fig. 18, the behavior of $\hat{\zeta}(z)$ for positive values of $x_{0}$ is sketched. By its definition, the diagram of $\hat{\zeta}(z)$ for a negative value of $x_{o}$ is exactly equal to the diagram of the correspondent positive value of $x_{o}$.

As can be seen from (44), the NDF depends only on the wavenumber $\beta=\frac{2 \pi}{\lambda}$, and on the geometrical parameters of the configuration $a, x_{o}, z_{\min }, z_{\max }$ which are related to the

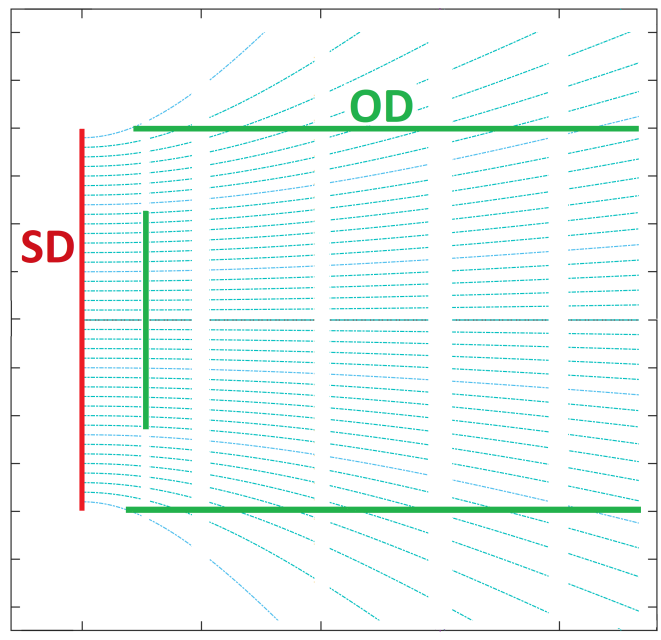

FIGURE 19. Geometry with combined observation domain (1 line parallel, and 2 lines orthogonal to the source).

size of the source, the position of the observation domain, and its extension.

Note that if $\zeta\left(z_{\min }\right)$ and $\zeta\left(z_{\max }\right)$ remain unchanged and $x_{o}$ changes in the set $x_{o} \in[a, \infty]$ then the NDF remains unchanged. Differently, if $\zeta\left(z_{\min }\right)$ and $\zeta\left(z_{\max }\right)$ remain unchanged and $x_{o}$ changes in the set $x_{o} \in[0, a]$ then the NDF increases with respect to $x_{o}$.

Let us remark that if the observation domain was infinite, the difference $\hat{\zeta}\left(z_{\min }\right)-\hat{\zeta}\left(z_{\max }\right)$ would be equal to 1 ; accordingly, the NDF would be at maximum $(\beta a) / \pi$. Since in practical cases the observation domain is necessarily finite, the NDF is always less than $(\beta a) / \pi$. Bearing in mind that the set of all possible currents is well represented with $(2 \beta a) / \pi$ independent functions, it follows that it is not possible to retrieve the current with data collected only on 1 line orthogonal to the source.

In order to retrieve the current, the data may be collected on 2 scanning lines of infinite extension placed along the axis $x_{o}=a$ and $x_{o}=-a$. However, since the observation domains are necessarily limited, also a little observation domain parallel to the source must be added (see Fig. 19). In such a case, the NDF of the radiated field can be derived by considering the result on the NDF provided here and that for an observation domain parallel to the source shown in [38].

\section{CONCLUSION}

In this paper, with reference to a magnetic strip current, an analytical expression of the NDF of the radiated field over a truncated line parallel to the source has been provided.

Since our study works in near zone for each value of $x_{o}$, it extends the results obtained for an observation domain centered with respect to the source located in Fresnel zone [31] or in near zone [32].

Let us point out that although the analysis has been developed in the case of a magnetic current, the expression of the NDF of the radiated field remains unchanged for an electric 
current. Indeed, in the case of an electric current, the kernel of the radiation operator $\mathcal{T}$ differs by that in (1) only for the amplitude but not for the phase (which represents the quantity that really affects the NDF). Accordingly, a similar analysis can be repeated by achieving the same result.

Before concluding, it is worth noting that this study, and that developed in [38] represent the mathematical basis for addressing configurations where the observation domain is a generic curve. The latter are very interesting in applications since a generic curve of observation may represent the trajectory followed by an UAV-based system for in-situ evaluation of radiating systems [39]-[43]. More in detail, the analysis made in [38] for an observation domain parallel to the source is suitable to study also the case where the observation domain is a smooth curve slowly varying (in such case no stationary points appear in the kernel of the integral equation involved in the computation of the singular values). Instead, the analysis developed here for an observation domain orthogonal to source allows to study also configurations where the observation domain is a smooth curve rapidly varying (in such case a stationary point appears in the kernel of the integral equation for the computation of the singular values).

\section{APPENDIX A}

\section{THE TRANSFORMATION, ITS INVERSE, AND THE} DERIVATIVE OF THE INVERSE FOR THE CASE $\mathrm{x}_{\mathrm{o}}>\mathrm{a}$ In this appendix, with reference to the the transformation

$$
\zeta(z)=\frac{1}{2 a}\left(\sqrt{\left(x_{o}+a\right)^{2}+z^{2}}-\sqrt{\left(x_{o}-a\right)^{2}+z^{2}}\right)
$$

used for $x_{o}>a$, the expression of $z(\zeta)$ and $\frac{d z}{d \zeta}$ is provided. In particular, the inverse function $z(\zeta)$ is given by

$$
z(\zeta)=\sqrt{\left(1-\zeta^{2}\right)\left(\frac{x_{o}^{2}}{\zeta^{2}}-a^{2}\right)}
$$

while its derivative is provided by

$$
\frac{d z}{d \zeta}=\frac{a^{2} \zeta-x_{o}^{2} \zeta^{-3}}{z(\zeta)}
$$

\section{APPENDIX B}

\section{APPROXIMATION OF THE AMPLITUDE TERMS}

$$
\frac{z\left(\zeta_{o}\right) f_{a}\left(\zeta_{o}, \zeta\right)}{\phi_{a}^{\prime}\left(\zeta_{o}\right), \zeta} \text { AND } \frac{z\left(\zeta_{o}\right) f_{-a}\left(\zeta, \zeta_{o}\right)}{\phi_{-a}^{\prime}\left(\zeta, \zeta_{o}\right)}
$$

In this appendix, it is shown how to derive the approximation of the amplitude terms $z\left(\zeta_{o}\right) \frac{f_{a}\left(\zeta_{o}, \zeta\right)}{\phi_{a}^{\prime}\left(\zeta_{o}, \zeta\right)}$ and $z\left(\zeta_{o}\right) \frac{f_{-a}\left(\zeta_{o}, \zeta\right)}{\phi_{-a}^{\prime}\left(\zeta_{o}, \zeta\right)}$ made in (14) and (15).

To obtain (14), the following approximation has been performed

$$
\frac{z_{o} f_{a}\left(z_{o}, z\right)}{\phi_{a}^{\prime}\left(z_{o}, z\right)} \approx \frac{\left.z_{o} f_{a}\left(z_{o}, z\right)\right|_{z_{o}=z}}{\left.\phi_{a}^{\prime}\left(z_{o}, z\right)\right|_{z_{o}=z}+\left.\frac{d \phi_{a}^{\prime}\left(z_{o}, z\right)}{d z_{o}}\right|_{z_{o}=z}\left(z_{o}-z\right)}
$$

Taking into account for the definitions of $f\left(x^{\prime}, z_{o}, z\right)$ and $\phi\left(x^{\prime}, z_{o}, z\right)$ provided in section III, it results that

$$
\begin{gathered}
\left.z_{o} f_{a}\left(z_{o}, z\right)\right|_{z_{o}=z}=\frac{z}{\left[\left(x_{o}-a\right)^{2}+z^{2}\right]^{3 / 2}} \\
\left.\phi_{a}^{\prime}\left(z_{o}, z\right)\right|_{z_{o}=z}=0 \\
\left.\frac{d \phi_{a}^{\prime}\left(z_{o}, z\right)}{d z_{o}}\right|_{z_{o}=z}=\frac{z\left(x_{o}-a\right)}{a\left[\left(x_{o}-a\right)^{2}+z^{2}\right]^{3 / 2}}
\end{gathered}
$$

Consequently, equation (44) can be rewritten as below

$$
\frac{z_{o} f_{a}\left(z_{o}, z\right)}{\phi_{a}^{\prime}\left(z_{o}, z\right)} \approx \frac{a}{\left(x_{o}-a\right)\left(z_{o}-z\right)}
$$

By exploiting the equation $z_{o}-z=\frac{d z}{d \zeta}\left(\zeta_{o}-\zeta\right)$, it results that

$$
\frac{z\left(\zeta_{o}\right) f_{a}\left(\zeta_{o}, \zeta\right)}{\phi_{a}^{\prime}\left(\zeta_{o}, \zeta\right)} \approx \frac{a}{\left(x_{o}-a\right)\left(\zeta_{o}-\zeta\right) \frac{d z}{d \zeta}} .
$$

The same discussion has been repeated also to approximate the term $z\left(\zeta_{o}\right) \frac{f_{-a}\left(\zeta_{o}, \zeta\right)}{\phi_{-a}^{\prime}\left(\zeta_{o}, \zeta\right)}$.

\section{APPENDIX C}

\section{THE TRANSFORMATION, ITS INVERSE, AND THE} DERIVATIVE OF THE INVERSE FOR THE CASE

$0 \leq x_{0} \leq a$

In this appendix, with reference to the the transformation

$$
\zeta(z)=\frac{1}{a+x_{o}}\left(\sqrt{\left(x_{o}+a\right)^{2}+z^{2}}-|z|\right)
$$

used for $0<x_{o}<a$, the expression of $z(\zeta)$ and $\frac{d z}{d \zeta}$ is provided. In particular, the inverse function $z(\zeta)$ has the following expression

$$
z(\zeta)=\frac{a+x_{o}}{2}\left(\frac{1}{\zeta}-\zeta\right)
$$

while its derivative is given by

$$
\frac{d z}{d \zeta}=-\frac{a+x_{o}}{2}\left(\frac{1}{\zeta^{2}}+1\right) .
$$

\section{APPENDIX D \\ APPROXIMATION OF THE TERM $\frac{f_{x_{o}}\left(\zeta_{o}, \zeta\right)}{\sqrt{\left|\phi_{x_{o}}^{\prime \prime}\left(\zeta_{o}, \zeta\right)\right|}}$}

In this appendix, the approximation (26) is justified.

Taking into account for the definitions of $f\left(x^{\prime}, z_{o}, z\right)$ and $\phi\left(x^{\prime}, z_{o}, z\right)$ provided in section III, it results that

$$
\frac{f_{x_{o}}\left(z_{o}, z\right)}{\sqrt{\left|\phi_{x_{o}}^{\prime \prime}\left(z_{o}, z\right)\right|}}=\frac{1}{z z_{o}} \frac{\sqrt{a}}{\sqrt{\left|z_{o}-z\right|}}
$$

Since $z_{o}-z=\frac{d z}{d \zeta}\left(\zeta_{o}-\zeta\right)$, equation (55) can be rewritten as below

$$
\frac{f_{x_{o}}\left(\zeta_{o}, \zeta\right)}{\sqrt{\left|\phi_{x_{o}}^{\prime \prime}\left(\zeta_{o}, \zeta\right)\right|}}=\frac{1}{2 z(\zeta) z\left(\zeta_{o}\right)} \frac{2 \sqrt{a}}{\sqrt{\left|\frac{d z}{d \zeta}\right|} \sqrt{\left|\zeta_{o}-\zeta\right|}}
$$


By performing the following approximation

$$
\frac{1}{\zeta_{o}-\zeta} \approx \frac{2}{\sqrt{\left|\zeta_{o}-\zeta\right|}} \operatorname{sign}\left(\zeta_{o}-\zeta\right)
$$

it results that

$$
\frac{f_{x_{o}}\left(\zeta_{o}, \zeta\right)}{\sqrt{\left|\phi_{x_{o}}^{\prime \prime}\left(\zeta_{o}, \zeta\right)\right|}} \approx \frac{\sqrt{a}}{2 z(\zeta) z\left(\zeta_{o}\right)} \frac{\operatorname{sign}\left(\zeta_{o}-\zeta\right)}{\sqrt{\left|\frac{d z}{d \zeta}\right|}\left(\zeta_{o}-\zeta\right)}
$$

\section{REFERENCES}

[1] G. Schnattinger, and T. Eibert, "Solution to the full vectorial 3D inverse source problem by multilevel fast multipole method inspired hierarchical disaggregation," IEEE Trans. Antennas Propag., vol. 60, no. 7, pp. 33253335, 2012.

[2] L. J. Foged, L. Scialacqua, F. Saccardi, J. L. A. Quijano, G. Vecchi, and M. Sabbadini, "Practical Application of the Equivalent Source Method as an Antenna Diagnostics Tool," IEEE Antennas Propag. Mag., vol. 54, no. 5, pp. 243-249, Oct. 2012.

[3] E.A. Marengo, and A.J. Devaney, "The inverse source problem of electromagnetics: Linear inversion formulation and minimum energy solution," IEEE Trans. Antennas Propag., vol. 47, pp. 410-412, 1999.

[4] C. Cappellin, A. Frandsen, and O. Breinbjerg, "Application of the SWE to-PWE Antenna Diagnostics Technique to an Offset Reflector Antenna", IEEE Trans. Antennas Propag. Mag., vol. 50, no. 5, pp. 204-213, Oct 2008.

[5] R.A.M. Mauermayer, Y. Weitsch, and T.F. Eibert, "Electromagnetic Field Synthesis by Hierarchical Plane Wave-Based Field Transformation," IEEE Trans. Antennas Propag., vol. 63, pp. 5561-5572, 2015.

[6] J. L. Araque Quijano, and G. Vecchi, "Improved-Accuracy Source Reconstruction on Arbitrary 3D Surfaces," IEEE Antennas Wirel. Propag. Lett., vol. 8, pp. 1046-1049, 2009.

[7] Y. Alvarez, F. Las-Heras, B. A. Dominguez-Casas, and C. Garcia, "Antenna Diagnostics Using Arbitrary-Geometry Field Acquisition Domains," IEEE Antennas Wirel. Propag. Lett., vol. 8, pp. 375-378, 2009.

[8] J. Kornprobst, R.A.M. Mauermayer, O. Neitz, J. Knapp, and T. Eibert, "On the Solution of Inverse Equivalent Surface-Source Problems" Prog. Electromagn. Res., vol. 165, pp. 47-65, 2019.

[9] K. Persson, M. Gustafsson and G. Kristensson, "Reconstruction and Visualization of Equivalent Currents on a Radome Using an Integral Representation Formulation", Progress in Electromagnetics Research B, vol. 20 , pp. $65-90,2010$.

[10] A.J. den Dekker, and A. van den Bos, "Resolution: a survey," J. Opt. Soc Am. A, vol. 14, no. 3, pp. 547-557, 1997.

[11] G.T Di Francia, "Degrees of freedom of an image," J. Opt. Soc. Am. A vol. 59, no. 7, pp. 799-804, 1969.

[12] R. Piestun, and D.A.B. Miller, "Electromagnetic degrees of freedom of an optical system," J. Opt. Soc. Am. A, vol. 17, pp. 892-902, 2000.

[13] D.A.B. Miller, "Waves, modes, communications, and optics: a tutorial," Advances in Optics and Photonics, vol. 11, no. 3, pp. 679-825, 2019.

[14] O. M. Bucci, G. Franceschetti, "On the degrees of freedom of scattered fields," IEEE Trans. on Antennas Propag., vol. 37, no. 7, pp. 918-926, July 1989.

[15] B. Fuchs, and A. G. Polimeridis, "Reduced Order Models for Fast Antenna Characterization," IEEE Trans. Antennas Propag., vol. 67, no. 8, pp. 56735677, Aug. 2019.

[16] K. Xu, L. Ran, Y. Zhong, and X. Chen, "Singular value decomposition of the current-to-field operator in solving inverse scattering problems," 2014 IEEE Antennas and Propagation Society International Symposium (APSURSI), Memphis, TN, 2014, pp. 659-660.

[17] David G. Fischer, Richard A. Frazin, Marius Asipauskas, and P. Scott Carney, "Information content of the near field: three-dimensional samples," $J$. Opt. Soc. Am. A, vol. 28, pp. 296-306, 2011.

[18] G. Leone, M.A. Maisto, R. Pierri, "Inverse Source of Circumference Geometries: SVD Investigation Based on Fourier Analysis," Prog. Electromagn. Res. $M$, vol. 76, pp. 217-230, 2018.

[19] T. Yin, Z. Wei and X. Chen, "Non-Iterative Methods Based on Singular Value Decomposition for Inverse Scattering Problems," IEEE Trans. on Antennas Propag., vol. 68, no. 6, pp. 4764-4773, Jun. 2020.

[20] G. Gennarelli, I. Catapano, F. Soldovieri and R. Persico, "On the Achievable Imaging Performance in Full 3-D Linear Inverse Scattering," IEEE Trans. on Antennas Propag., vol. 63, no. 3, pp. 1150-1155, March 2015.
[21] T. Isernia, G. Leone, R. Pierri "Sulle dimensioni essenziali dei campi: un approccio ai valori singolari," IX Riunione Nazionale di Elettromagnetismo, Assisi, Itay, 1992.

[22] R. Somaraju, and J. Trumpf, "Degrees of freedom of a communication channel: using DOF singular values," IEEE Trans. Inf. Theory, vol. 56, pp 1560-1573, 2010.

[23] R. Pierri, and F. Soldovieri, "On the information content of the radiated fields in the near zone over bounded domains," Inverse Problems, vol. 14 no. 2, pp. 321-337, 1998.

[24] M. A. Maisto, R. Solimene, and R. Pierri, "Resolution limits in inverse source problem for strip currents not in Fresnel zone," J. Opt. Soc. Am. A vol. 36, pp. 826-833, 2019.

[25] G. Leone, F. Munno and R. Pierri, "Inverse Source on Conformal Conic Geometries," IEEE Trans. Antennas Propag., 2020.

[26] A. S. Y. Poon, R. W. Brodersen, and D. N. C. Tse, "Degrees of freedom in multiple-antenna channels: a signal space approach," IEEE Trans. Inf. Theory, vol. 51, no. 2, pp. 523-536, Feb. 2005.

[27] A. Capozzoli, C. Curcio, A. Liseno, "Multi-frequency planar near-field scanning by means of singular-value decomposition (SVD) optimization," IEEE Antennas Prop. Mag., vol. 53, no. 6, pp. 212-221, Dec. 2011.

[28] M. A. Maisto, G. Leone, A. Brancaccio, R. Solimene, "Efficient Planar Near-field Measurements for Radiation Pattern Evaluation by a Warping Strategy," IEEE Access, 2021.

[29] J. Ranieri, A. Chebira, and M. Vetterli, "Near-Optimal Sensor Placement for Linear Inverse Problems," IEEE Trans. Signal Process., vol. 62, no. 5 , pp. 1135-1146, March 2014.

[30] C. Jiang, Y. Soh, and H. Li "Sensor placement by maximal projection on minimum eigenspace for linear inverse problems," IEEE Trans. Signal Process., vol. 64, pp. 5595-5610, 2016.

[31] R. Pierri, A. Liseno, F. Soldovieri, and R. Solimene, "In-depth resolution for a strip source in the Fresnel zone," J. Opt. Soc. Am. A, vol. 18, no. 2 , pp. 352-359, 2001.

[32] M.A. Maisto, R. Solimene, and R. Pierri, "Depth resolution in strip curren reconstructions in near non-reactive zone," J. Opt. Soc. Am. A, vol. 36, no. 6, pp. 975-982, 2019

[33] R. Moretta, R. Pierri, "Performance of phase retrieval via phaselift and quadratic inversion in circular scanning case," IEEE Trans. Antennas Propag., vol 67, no. 12, pp. 7528-7537, 2019.

[34] D. Slepian, and H.O. Pollack, "Prolate spheroidal wave functions, Fourier analysis, and uncertainty-I," The Bell System Technical Journal, vol. 40, no. 1 , pp. 43, 1961.

[35] K. Khare, and N. George, "Sampling theory approach to prolate spheroida wavefunctions," Journal of Physics A: Mathematical and General, vol. 36 , no. 39, pp. 10011, 2003.

[36] M. Bertero, and P. Boccacci. Introduction to Inverse Problems in Imaging; IOP Publishing: Bristol, UK, 1998.

[37] N. Bleistein, R.A. Handelsman. Asymptotic Expansions of Integrals Dover Publications: New York, NY, USA, 1986.

[38] R. Pierri, and R. Moretta, "Asymptotic study of the radiation operator for the strip current in near zone" Electronics, vol. 9, no. 6, art. no. 911, 2020

[39] G. Virone et al., "Antenna pattern verification system based on a micro unmanned aerial vehicle (UAV)", IEEE Antennas Wireless Propag. Lett. vol. 13, pp. 169-172, 2014

[40] T. Fritzel, R. Straub, H.J. Steiner, C. Eisner, and T. Eibert, "Introduction into an UAV-based near-field system for in-situ and large-scale antenna measurements," IEEE Conference on Antenna Measurements \& Applications (CAMA), pp. 1-3, 2016.

[41] M. G. Fernandez et al. "Antenna Diagnostics and Characterization using Unmanned Aerial Vehicles," IEEE Access, vol. 5, pp. 23563-23575, 2017.

[42] G. Virone et al. "Strong Mutual Coupling Effects on LOFAR: Modeling and In Situ Validation," IEEE Trans. Antennas Propag., vol. 66, no. 5, pp 2581-2588, 2018.

[43] L. Ciorba et al. "Near-Field Phase Reconstruction for UAV-based Antenna Measurements," 13th European Conference on Antennas and Propagation (EuCAP), 2019. 


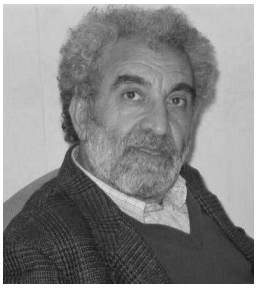

ROCCO PIERRI received the Laurea degree (summa cum laude) in electronic engineering from the University of Naples "Federico II" in 1976.

He was a Visiting Scholar with the University of Illinois at Urbana-Champaign, Urbana, IL, USA; Harvard University, Cambridge, MA, USA; Northeastern University, Boston, MA, USA; Supelec, Paris, France; and the University of Leeds, Leeds, U.K. He also extensively lectured abroad in many universities and research centers.

He is currently a Full Professor with the University of Campania "Luigi Vanvitelli", Aversa, Italy.

His current research interests include antennas, phase retrieval, near-field techniques, inverse electromagnetic scattering, subsurface sensing, electromagnetic diagnostics, microwave tomography, inverse source problems, and information content of radiated field.

Prof. Pierri was a recipient of the 1999 Honorable Mention for the $\mathrm{H}$. A. Wheeler Applications Prize Paper Award of the IEEE Antennas and Propagation Society.

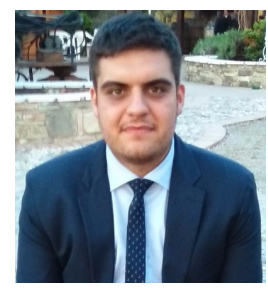

RAFFAELE MORETTA received the M.S. degree (summa cum laude) in electronic engineering from the University of Campania "Luigi Vanvitelli" in 2018, where he is currently pursuing the Ph.D. Degree.

Since 2016, he has started scientific cooperation with the Electromagnetic Fields Group of the University of Campania "Luigi Vanvitelli".

His current research interests include inverse problems in elec tromagnetics with particular attention to phase retrieval and near field techniques.

Dr. Moretta is a student member of the Institute of Electrical and Electronics Engineers (IEEE) and of the Italian Society of Electromagnetism (SIEM) Moreover, he was under consideration by the committee of the IEEE Antennas Propagation Society for the R.W.P. King Award 2020. 